\title{
$\alpha$-GeTe and (GeMn)Te Semiconductors: a New Paradigm for Spintronics
}

\author{
J. Krempaský ${ }^{1, a)}$, G. Springholz ${ }^{2}$, J. Minár ${ }^{3}$ and J. H. Dil ${ }^{4}$ \\ ${ }^{I}$ Photon Science Division, Paul Scherrer Institut, 5232 Villigen PSI, Switzerland \\ ${ }^{2}$ Institut fur Halbleiter-und Festkörperphysik, Johannes Kepler Universität, 4040 Linz, Austria \\ ${ }^{3}$ University of West Bohemia New Technologies - Research Centre, Univerzitni 8, 30614 Plzeň, Czech Republic \\ ${ }^{4}$ Institute of Physics, École Polytechnique Fédérale de Lausanne, 1015 Lausanne, Switzerland \\ ${ }^{a)}$ Corresponding author: juraj.krempasky@psi.ch
}

\begin{abstract}
GeTe is the simplest known binary ferroelectric semiconductor with a narrow band gap. Below $700 \mathrm{~K}$ it assumes a non-centrosymmetric rhombohedral structure in which an electric dipole is formed due to a relative $\mathrm{Ge} / \mathrm{Te}$ sublattice displacement along the [111] direction. Ferroelectric polarization results from asymmetric positions of Ge and Te atoms along that direction and ensures that the system possess a well-defined axis for symmetry breaking, resulting in a giant Rashba-type spin splitting of the bulk band structure. We report on first-principle calculations which indicate that the large lattice distortion responsible for the ferroelectric order is also the most significant ingredient for the giant Rashba-type spin-splitting. We review the experimental verification of this giant Rashba-type splitting and show the main results proving that $(\mathrm{GeMn}) \mathrm{Te}$ is a new paradigm multiferroic semiconductor with magnetoelectic properties, offering broad opportunities for spintronics materials design.
\end{abstract}

\section{$\alpha$-GeTe FERROELECTRIC RASHBA SEMICONDUCTOR}

GeTe is a ferroelectric semiconductor that recently attracted interest because of very large Rashba-type spin splitting of the bulk band structure [1,2,3]. Ferroelectric polarization is related to an asymmetric position of Ge layers between Te layers along the [111] direction ( $\alpha-G e T e)$. Spin-orbit coupling (SOC) effects in materials with broken inversion symmetry are responsible for peculiar spin textures, in a generic sense referred to as Rashba-type effects. In $\alpha$-GeTe the sizable spin orbit coupling and inversion symmetry breaking provided by the remanent ferroelectric polarization constitutes an intriguing route for a non-volatile electrical control of the spin degrees of freedom. Without consideration of surface effects it is expected that a reversal of the Rashba-type spin polarization is coupled to reversal of the ferroelectric polarization, since this corresponds to reversing of the crystal asymmetry.

In order to verify the Rashba-type splitting in GeTe we performed spin- and angle-resolved photoelectron spectroscopy (SARPES) measurements. The measurements were performed at the Swiss Light Source of the Paul Scherrer Institut. The SARPES data were measured with the Mott polarimeters at the COPHEE end station at $20 \mathrm{~K}$ [4]. Figure 1a shows a schematic representation of the $\alpha$-GeTe surface electronic structure near the $Z$ point where the Rashba-type splitting is most pronounced [3]. However, near the Z-point it is also shadowed by surface resonances denoted with black dashed lines in Fig. 1a [3,5]. It turns out that the $\alpha$-GeTe bulk-representative bands, denoted in red and blue in Fig. 1a, are best observed beneath the Dirac point along the Z-U direction for binding energies around $0.5 \mathrm{eV}$. This locus of energy and momenta is indicated by a green dashed line in Fig 1a. In this work we briefly summarize the magnitude of the spin splitting and the peculiar spin texture of $\alpha$-GeTe bulk-representative states, and explore their respective origin.

Applied Physics of Condensed Matter (APCOM 2018)

AIP Conf. Proc. 1996, 020026-1-020026-6; https://doi.org/10.1063/1.5048878

Published by AIP Publishing. 978-0-7354-1712-0/ $\$ 30.00$ 

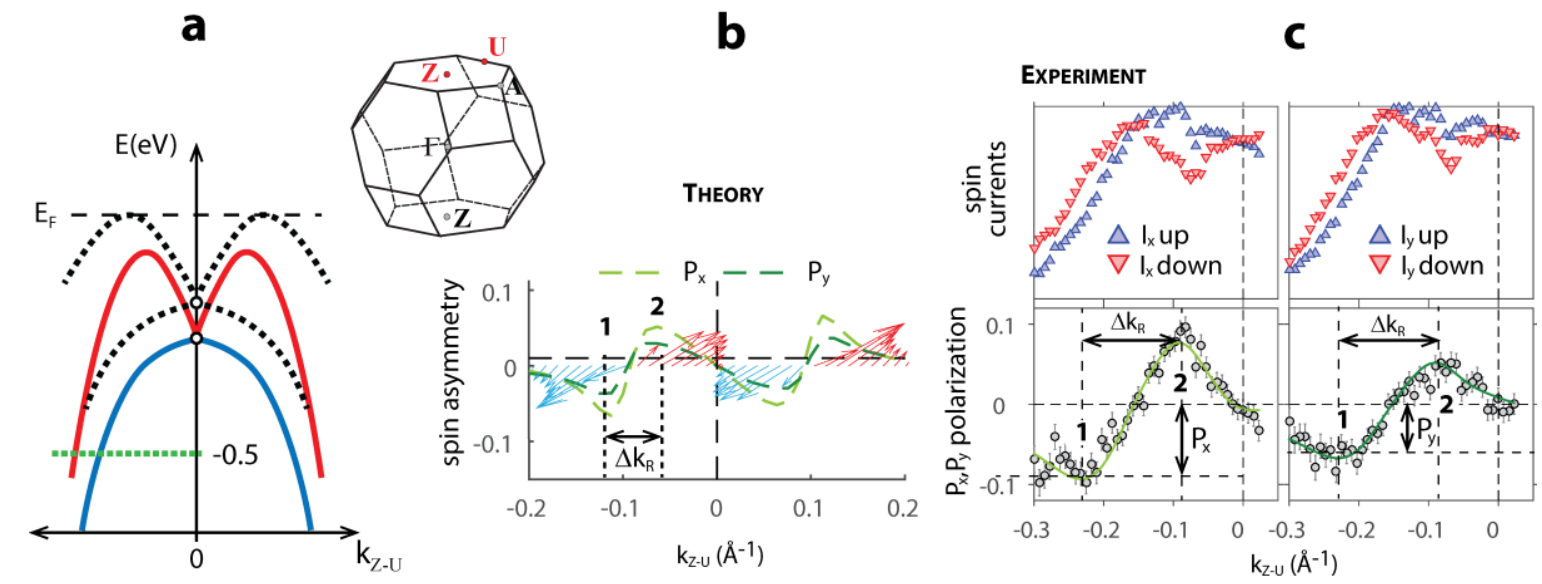

FIGURE 1. (a) Schematic representation of the $\alpha$-GeTe surface electronic structure measured near the $Z$-point $(h v=22 \mathrm{eV})$ along the ZU direction (see Brillouin zone inset). (b) Calculated in-plane spin-asymmetry along the U-Z-U direction. (c) SARPES spin in-plane currents (top panels) and polarizations (lower panels) including data fits (green lines), measured at a binding energy of $0.5 \mathrm{eV}$ along the $\mathrm{Z}-\mathrm{U}$ path indicated in (a) by dashed green line. $\Delta k_{R}$ is the Rashba-type momentum splitting (see text).

Fig. 1b-c visualizes theoretical and experimental data as spin-resolved momentum distribution curves (MDC). For the theoretical results we employed self-consistent electronic structure calculations performed within the $a b$ initio framework of spin-density functional theory by use of the Vosko et al. parameterization of the exchange and correlation potential [6]. The electronic structure was calculated within the relativistic multiple-scattering or Korringa-Kohn-Rostoker (KKR) formalism [7]. These self-consistent calculations have been performed for bulk GeTe as well as for semi-infinite surface of $\alpha$-GeTe within so called tight-binding KKR method. The corresponding ground state band structures are presented in terms of Bloch spectral functions. To theoretically analyze the photoemission process, one-step model photoemission calculations for the semi-infinite surface were performed. This fully relativistic approach is based on the spin-density matrix formulation for photocurrent and it includes all matrix-element effects, multiple scattering in the initial and final states, and all surface-related effects in the excitation process [8]. The resulting theoretical representation of the $\alpha-G e T e$ surface electronic structure is summarized in the cartoon in Fig.1a.

For both SARPES measurements and calculations the sample was aligned in such a way that $P_{x}\left(P_{y}\right)$ spin polarization is along the Z-A (Z-U) direction, as indicated in the Brillouin zone in Fig. 1a. The calculations predict bulk states with distinct canted helicity for in-plane polarization $\left(P_{x} \approx 2 P_{y}\right)$, as indicated by the green dashed lines in panel (b). The corresponding experimental data summarized in panel (c) also identify the peaks $1-2$ as bulk Rashba-split bands with a splitting $\Delta k_{R} \approx 0.12 \AA^{-1}$ and similar $P_{x} \approx 2 P_{y}$ spin polarization ratio. In order to illustrate the in-plane spin polarization $P_{x y}$, MDC data is visualized in a quiver plot to indicate the marked spin canting of the two Rashba-split bands 1-2 indicated by red and blue arrows. We emphasize that both the magnitude of $P_{x}, P_{y}$ spin polarizations, and spin splitting $\Delta k_{R}$ are in agreement with the ground state calculations, thus confirming the characteristic canted spin texture of the $\alpha$-GeTe bulk bands.

\section{Mechanism Responsible for $\alpha$-GeTe Giant Rashba-Type Splitting}

In a simplified approach the strength of the Rashba splitting can be quantified through the momentum shift $\Delta k_{R}$ indicated in Fig. 1b-c and related change in energy. For GeTe this Rashba strength parameter $\alpha_{R}$ is as high as $4.2 \mathrm{eV} \AA$ [3]. This is a giant value which surpasses materials so far studied on surfaces or interfaces where the inversion symmetry is intrinsically broken. We recall that the Rashba effect appears when the structural inversion symmetry is broken in a presence of spin-orbit coupling (SOC). This lifts the spin degeneracy of the states and was first described for a two-dimensional electron gas with structural inversion asymmetry [9]. 

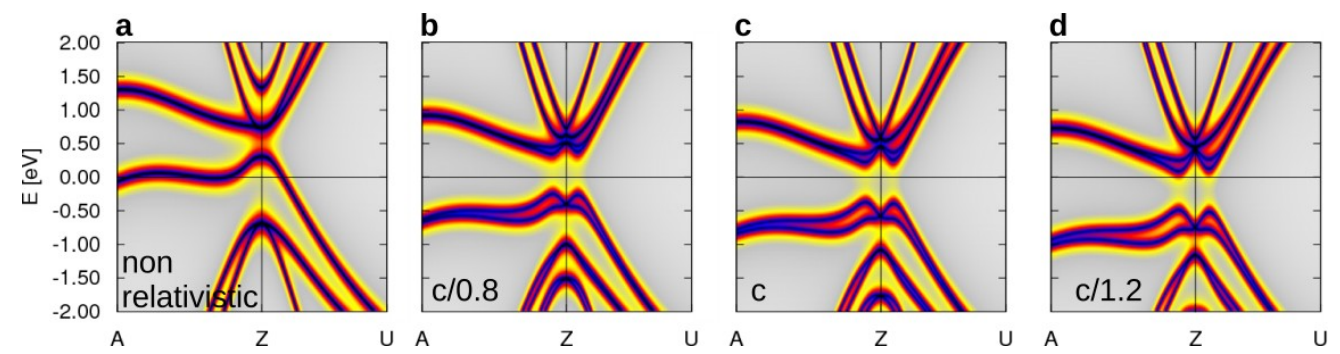

FIGURE 2. Bloch spectral functions for distorted GeTe lattice under variable strength of spin-orbit interaction: (a) Nonrelativistic $\alpha$-GeTe band structure. (b-d) Band structure SOC manipulations by tuning the speed of light $c$ within the fully relativistic Dirac equation based KKR method.

The fact that Rashba effects requires both effects to be present raises question whether symmetry breaking or SOC is responsible for the giant $\alpha_{R}$ parameter in GeTe. To address this question, Figure 2 shows how the $\alpha$-GeTe band maps vary under SOC manipulations within the fully relativistic (Dirac equation based) KKR method. As it was already pointed out by D. Di Sante et al. [1], by manipulating the ferroelectric order parameter it was shown that for a non-distorted $\alpha$-GeTe lattice $(\mathrm{NaCl})$ the spin-split Rashba states along AZU-direction are degenerate. Let us now keep the lattice distortion, i.e. ferroelectric order, and manipulate the strength of the SOC as summarized in Fig. 2. By turning off all relativistic effects including the SOC (panel a), the band map is degenerate as in the case of a non-distorted lattice [1]. By scaling of the speed of the light in the Dirac equation, panels b-d indicate that the Rashba parameter $\alpha_{R}$ displays only marginal increase even in the super-relativistic limit (panel d). We note that the SOC term in the Dirac equation scales with $1 / c^{2}$, which means that by increasing the relativistic speed by $20 \%$ the SOC increases by $250 \%$. But even under such super-relativistic condition there is no significant change in $\alpha_{R}$, hence the giant Rashba-type splitting is to be attributed to non-centrosymmetric arrangement of the Ge and Te atoms responsible for the ferroelectric order.

\section{$\alpha-G e T e$ Rashba-Type Spin Texture}

$\alpha$-GeTe has spin-polarized topologically trivial bulk bands with the spin chirality coupled to the FE polarization. As dictated by Rashba physics, the spin texture above and below the Dirac point must be different. Between the Dirac point and the band apex the Rashba-split states have the same helicity, whereas below the Dirac point they have opposite helicity [10]. Such a spin texture comparing theoretical (dashed lines) and experimental data (arrows and green solid lines) near the Fermi level and at $0.3 \mathrm{eV}$ binding energy is presented in Fig. 3. Finally, Fig. 3a shows the overall spin texture associated with ARPES band maps, which is indicated by the spin vectors measured near $k= \pm 0.1 \AA^{-1}$ along both Z-A and Z-U directions near the Fermi level and below the Dirac point. For clarity A-D labels refer to the ARPES energy and momenta with corresponding spin-texture indicated in panels (a) and (c). We note that the spin texture above and below the Dirac point is indeed different with co-chiral or opposite helicity, respectively, and that all data show pronounced and consistent spin canting both in theory and experiment, as already discussed above.

A closer look at the ARPES band map in Fig. $3 \mathrm{~b}$ shows that the bulk Rashba bands indicated by red-blue lines are not resolved, in particular the outer Rashba band indicated in red. As already mentioned, this is due to the fact that the $\alpha$-GeTe bulk bands are shadowed by surface resonances (dashed lines in Fig. 1a; [3]) and thus difficult to resolve in ARPES. However by measuring the same SARPES data for a lower binding energy around $-0.5 \mathrm{eV}$ (Fig.1), the spectral weight of the surface resonances is suppressed and thus both Rashba bands 1-2 along the green dashed cut indicated in Fig. 1a are resolved. We note that despite the low spectral resolution of the electron analyzer in spin-integrated ARPES, it is a common practice that the Rashba bands get resolved in SARPES as a combination of four measured experimental quantities: $P_{x}, P_{y}, P_{z}$ spin polarizations and total photoemission signal from the two Mott polarimeters. In such case the spin "tag" in 3D SARPES data is unambiguously resolving the $\alpha-\mathrm{GeTe}$ Rashba-type splitting, in agreement with high resolution ARPES band maps in which the $\alpha$-GeTe surface electronic structure depicted in Fig. 2f was confirmed [3]. 
a

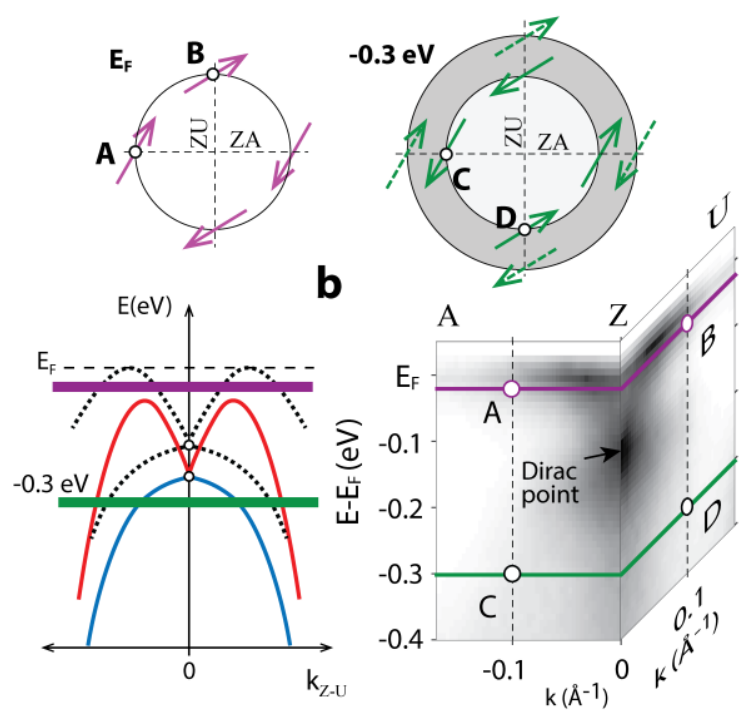

C

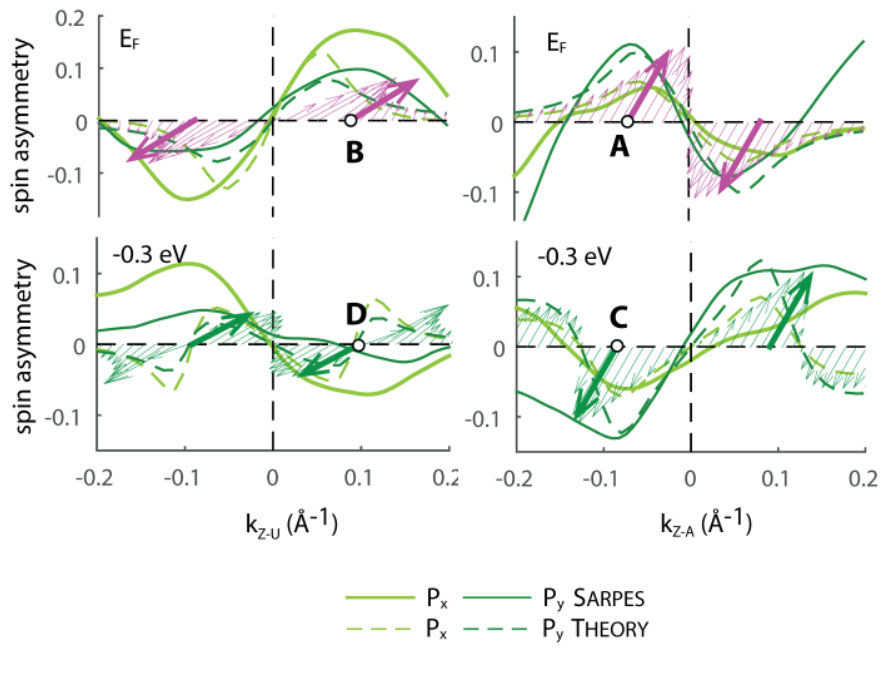

FIGURE 3. (a) Theoretical spin texture for $\alpha$-GeTe near Fermi level ( $E_{F}$, magenta) and at -0.3 eV binding energy (green), dashed green arrows refer to outer Rashba-type bands below the Dirac point, experimentally resolved in SARPES for lower binding energy (Fig. 1c). (b) Simplified $\alpha$-GeTe band model showing surface resonances indicated by black dashed lines, and bulk Rashba bands denoted with red and blue solid lines (from Ref.[11]); compared to ARPES data measured at $h v=22 \mathrm{eV}$ along ZA and ZU directions. (c) Comparison between theoretical (dashed lines) and experimental SARPES MDC data (solid lines) which map the in-plane spin polarization of the bulk Rashba bands. For clarity, the energy and momenta indicated by labels A-D in panel (b) are also indicated in panels (a) and (c). The spin texture in panel (a) summarizes the orientation of theoretical spin vectors indicated with thick arrows in panel (c), dashed green spins are not resolved in SARPES data (see text).

Having localized the $\alpha$-GeTe bulk Rashba-type bands, following the concept proposed by D. Di Sante et al. [1], the reversal of the ferroelectric polarization should also revert the spin helicity. In order to demonstrate this, we performed operando SARPES measurements for $\alpha$-GeTe films grown on conductive InP substrates by using a top-gate structure to enable application of an electric field during SARPES measurement [11]. By monitoring the spin texture in ultra-high vacuum via SARPES, upon application of voltages across the film and enabling the detection of photoelectrons across an Au-grid placed on top of the sample surface, we found that the spin texture is indeed influenced and changes direction for opposite voltage polarity. We emphasize that the macroscopic control of the ferroelectric polarization in a highly conductive semiconductors such as $\alpha-\mathrm{GeTe}$ is challenging. For this reason the $\mathrm{Au}$ mesh was coated with insulating $\mathrm{Al}_{2} \mathrm{O}_{3}$ grid as top electrode and the measurements were made under applied bias voltage. Furthermore, the measured Rashba-type spin electric switching was accompanied with strong dependence on the applied field polarity, resulting in different magnitudes of spin rotation: first application of negative voltage results in a larger magnitude of spin manipulation compared to the pristine case; and conversely, first application of positive voltage results in smaller one. Moreover, subsequent manipulations of the spins were stalled due to ferroelectric fatigue effects, unless the system was re-juvenated by annealing to restore the ferroelectric order. Such observation proves the spin manipulation by electric field, but is far from a robust all-electric spin switching between remnant polarization states. Further improvements in domain stabilization and sample growth are needed to achieve this goal.

\section{(GeMn)Te MULTIFERROIC RASHBA SEMICONDUCTOR}

Allegedly, regarding the sensitivity of semiconductor properties to impurities, Wolfgang Pauli was not very keen on the idea to deal with them at all: "One shouldn't work on semiconductors that are a filth mess; who knows whether any semiconductors exist" [12]. We note that understanding the role of magnetic impurities in semiconductors is a much more complex issue even in the era of highly accurate molecular beam epitaxy techniques. But with regard to increasing interest in exploring the spin degree of freedom in semiconductors by the 
emerging field of semiconductor spintronics, magnetic impurities became a burgeoning field in condensed matter physics. In our case for moderate Mn-dopings $(\mathrm{Mn}<20 \%)$, (GeMn)Te becomes ferromagnetic while maintaining the ferroelectric properties [13]. This opens up new spin-based functionalities because multiferroicity entangles with the Rashba splitting in a three-dimensional system [14]. In this new class of materials, denoted as MUltiFErroic Rashba Semiconductors (MUFERS), we demonstrated for the first time that there is a Zeeman gap opening in the host Rashba $\alpha$-GeTe (Fig. 4a) as predicted by theory (Fig. 4b). By measuring the perpendicular spin polarization $p_{z}$ we demonstrated that the external magnetic fields enable switching of the bulk spin texture (Fig. 4d); and that the spin manipulation is also possible by electric fields (Fig. 4e). This means that the (GeMn)Te functional properties expand to all-electric control of magnetism as schematically shown in Fig. 4c. Single phase materials that show simultaneously ferroelectricity and ferromagnetism are very rare, but their entanglement with the switching of the Rashba-type splitting is to this date unique for (GeMn)Te. Thus, from an application viewpoint, (GeMn)Te fulfils all criteria for mutual control of magnetism and ferroelectricity via magnetoelectric coupling to Rashba physics. The system thus paves the way for novel multifunctional spin devices, expected to have great advantages with respect to their charge counterparts, in particular due to reduced energy consumption.

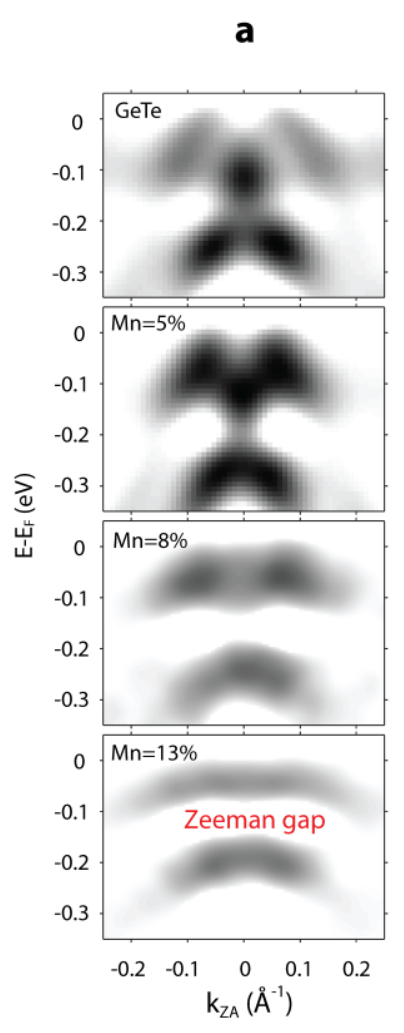

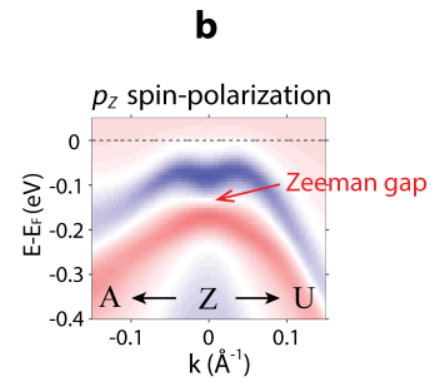

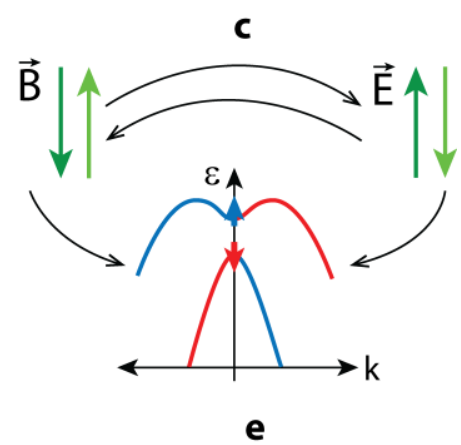

d

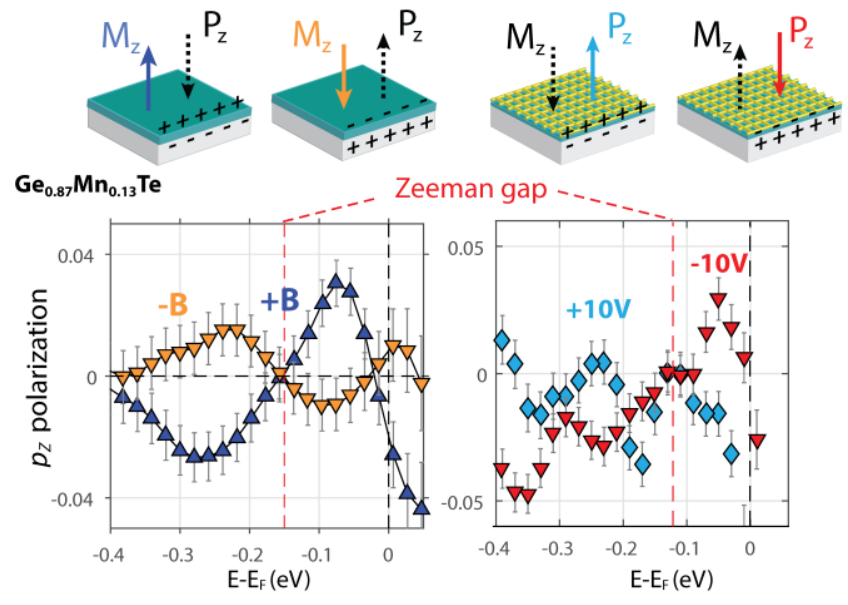

FIGURE 4. (a) ARPES data showing the Zeeman gap opening upon Mn-doping (from Ref.[14]). (b) Theoretical calculations for the perpendicular spin polarization $p_{z}$ for $\mathrm{Ge}_{0.87} \mathrm{Mn}_{0.13} \mathrm{Te}$ (from Ref.[14]). (c) Schematic view of the spin texture response to external magnetic or electric fields. (d) Manipulation of the $p_{z}$ spin polarization by magnetizing the sample in opposite directions (SARPES data were measured in remanence, from Ref.[14]). (e) Manipulation of the $p_{z}$ spin polarization by applied electric field (from Ref.[11]).

\section{ACKNOWLEDGMENTS}

This work was supported by the Swiss National Science Foundation Project PP00P2 144742 and PP00P2 170591, and the Austrian Science Funds Project P 30960. J. M. acknowledges the Project CEDAMNF (Project No CZ.02.1.01/0.0/0.0/15.003/0000358) of the Czech ministerium MSMT. 


\section{REFERENCES}

1. D. Di Sante et al., Advanced Materials 25, 509 (2013).

2. S. Picozzi, Frontiers in Physics 2 (2014).

3. J. Krempaský et al., Disentangling bulk and surface Rashba effects in ferroelectric $\alpha$-GeTe, PRB 94, 205111 (2016)

4. M. Hoesch et al., J. Electron Spectrosc. Relat. Phenom. 124, 263 (2002).

5. J. Krempaský et al., Spin-resolved electronic structure of ferroelectric $\alpha-G e T e$ and multiferroic $\mathrm{Ge}_{1-\mathrm{x}} \mathrm{Mn}_{\mathrm{x}} \mathrm{Te}$, Journal of Physics and Chemistry of Solids, 0022-3697, (2017)

6. $\quad$ Y. A. Bychkov, E. I. Rashba, J. Phys. C Solid State Phys. 17 (1984) 6039.

7. Vosko S. H, Wilk L. and Nusair M. 1980 Can. J. Phys. 581200

8. Ebert H., Ködderitzsch D. and Minár J. 2011 Rep. Prog. Phys. 74096501

9. Braun J., Minar J., Ebert H., 2018 Physics Reports 7401

10. F. Meier, V. Petrov, S. Guerrero, Ch. Mudry, L. Patthey, J. Osterwalder, and J. H. Dil, Unconventional Fermi surface spin textures in the $\mathrm{BixPb}_{1-\mathrm{x}} / \mathrm{Ag}(111)$ surface alloy, PRB 79, 241408(R) (2009)

11. J. Krempaský et al., Operando imaging of all-electric spin texture manipulation in ferroelectric and multiferroic Rashba semiconductors. Physical Review X XX XXX 2018

12. Out of the Crystal Maze: Chapters from the History of Solid-State Physics (eds. Hoddeson, L., Braun, E., Teichmann, J. \& Weart, S.), Oxford Univ. Press, Oxford, 1992.

13. H. Przybylińska et al., Magnetic-Field-Induced Ferroelectric Polarization Reversal in the Multiferroic $\mathrm{Ge}_{1-\mathrm{x}} \mathrm{Mn}_{\mathrm{x}} \mathrm{Te}$ Semiconductor, Phys. Rev. Lett. 112, 047202 (2014).

14. J. Krempaský et al., Entanglement and manipulation of the magnetic and spin-orbit order in multiferroic Rashba semiconductors, Nat. Commun. 7, 13071 (2016) 